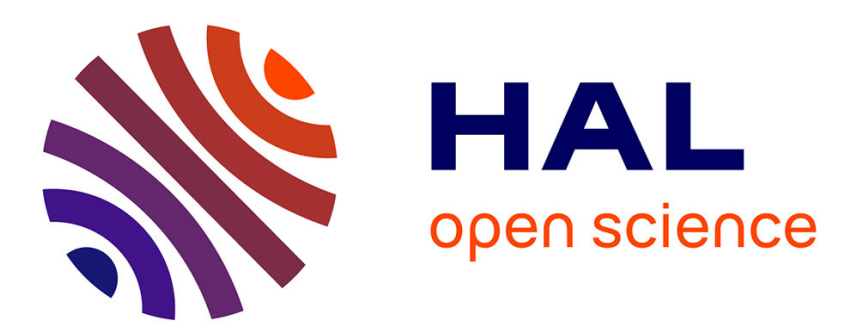

\title{
Étude de la structure détaillée des courbes de diffusion des ondes électromagnétiques par les sphères diélectriques
}

\author{
Jean Mevel
}

\section{- To cite this version:}

Jean Mevel. Étude de la structure détaillée des courbes de diffusion des ondes électromagnétiques par les sphères diélectriques. J. Phys. Radium, 1958, 19 (7), pp.630-636. 10.1051/jphysrad:01958001907063000 . jpa-00235906

\section{HAL Id: jpa-00235906 https://hal.science/jpa-00235906}

Submitted on 1 Jan 1958

HAL is a multi-disciplinary open access archive for the deposit and dissemination of scientific research documents, whether they are published or not. The documents may come from teaching and research institutions in France or abroad, or from public or private research centers.
L'archive ouverte pluridisciplinaire HAL, est destinée au dépôt et à la diffusion de documents scientifiques de niveau recherche, publiés ou non, émanant des établissements d'enseignement et de recherche français ou étrangers, des laboratoires publics ou privés. 


\title{
ÉTUDE DEİLA STRUCTURE DÉTAILLÉE \\ DES COURBES DE DIFFUSION DES ONDES ELLETROMAGNÉTIQUES PAR LES SPHËRES DIÉLECTRIQUES
}

\author{
Par Jean MEVEL,
}

Faculté des Sciences de Rennes.

\begin{abstract}
Résumé. - Des déterminations théoriques récentes de la courbe de diffusion de la lumière par les sphères diélectriques, ont montré qu'elle présente, superposée aux ondulations principales, une série d'indentations secondaires dont l'origine n'a pas été élucidée. Dans ce qui suit, nous établissons que cette structure est due aux résonances des termes multipolaires constituant le rayonnement diffusé et nous interprétons chaque maximum secondaire. Nous avons dû pour cela formuler un théorème nouveau permettant de déterminer aisément l'intensité d'excitation des modes, nous en déduisons les positions et les intensités des maxima de la structure détaillée. Enfin, nous montrons que la courbe de diffusion actuellement connue est imparfaite et nous indiquons de quelle manière on doit la modifier.
\end{abstract}

\begin{abstract}
Recent theoretical computations of the light extinction curve by dielectric spheres have shown that it has, superposed on the major oscillations, very rapid fluctuations of small amplitude : this feature has not been explained. In this work, we show that these fluctuations result from resonances of multipole terms which form the scattered radiation and we elucidate the origin of each of these secondary maxima. We demonstrate a new theorem which gives the amplitude of the multipole modes, and from this, we compute the positions and intensities of the detailed structure. Finally we show that the present known extinction curve is not correct and we indicate in what manner it must be modified.
\end{abstract}

Introduction. - Soit un faisceau lumineux plan, monochromatique, de longueur d'onde $\lambda$, qui se propage dans la direction $\mathrm{OZ}$ (fig. 1) et qui est

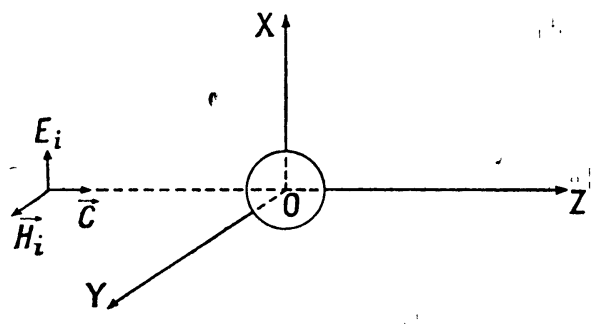

FIG. 1.

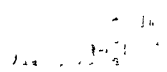

polarisé rectilignement, de sorte que le champ électrique incident soit parallèle à $\mathrm{OX}$. Plaçons au point $\mathrm{O}$ une sphère de rayon $a$ et d'indice $n^{\prime}$ quelconques : elle perturbe et disperse selon un processus complexe (diffraction, réflexion, réfraction) une portion $d u$ faisceau incident qui se trouve ainsi affaibli. L'étude qui suit se rapporte à la détermination de cet affaiblissement ; on ne considère pas la répartition de la lumière diffusée et on néglige tout effet quantique.

L'action de la sphère sur le faisceau incident est caractérisée par le "facteur de diffusion " $K(\alpha, n)$. c'est le rapport du flux lumineux total diffusé par la sphère dans toutes les directions, au flux incident sur sa section droite. Cette grandeur présentant un grand intérêt pour l'optique des milieux troubles, de nombreux auteurs se sont attachés à la déterminer théoriquement. Mie, le premier, en donna l'expression mathématique rigoureuse, elle est très complexe et le calcul numérique en est long et délicat. Lorsqu'on ne dispose que de machines à calculer classiques, les formules sont presque inutilisables pour des valeurs de $\alpha=2 \pi a / \lambda$. dépassant quelques unités. Des méthodes d'approximation dues à de nombreux chercheurs ont permis de compléter les renseignements fournis par la théorie de Mie et de déterminer la forme de la courbe de diffusion ( $f$ g. 2).

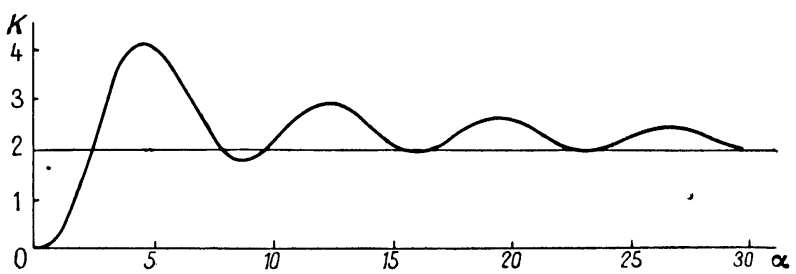

FIg. 2. - Courbe de diffusion (valeurs moyennes).

Une série de calculs entrepris par Penndorf [1], selon la théorie de Mie, à l'aide de calculatrices électroniques, montra que la structure indiquée ci-dessus n'était qu'approximative. La courbe obtenue, publiée pour la première fois en 1953 [2] présente une série d'indentations que les calculs précédents n'avaient pas montrée. Les caractéristiques essentielles qui résultent de l'examen de cette courbe sont les suivantes (fig 4) :

1) La valeur asymptotique $K=2$ : l'énergie interceptée par une grosse sphère est le double de celle qu'elle occulte géométriquement. 
2) L'existence de grandes fluctuations dont les maxima et les minima se placent approximativement à des valeurs fixes de $2 \alpha(n-1)$.

3) Le fait que la courbe est située en moyenne plus haut que son asymptote.

4) L'existence d'une structure détaillée.

Les trois premiers points ont été examinés par Van de Hulst [3] qui leur a trouvé des explications satisfaisantes, nous n'y reviendrons pas et nous nous attacherons ici à déterminer l'origine de la structure détaillée (“ Ripple » de Van de Hulst).

Rappel des résultats essentiels de la théorie de Mie. - Avec les seules hypothèses de l'existence du champ et de la vérification des équations de Maxwell, Mie [4] a pu établir les expressions rigoureuses du problème de diffraction des ondes électromagnétiques par les sphères. Nous renvoyons pour le détail de la théorie au travail original de Mie et aux exposés plus récents qui en ont été publiés $[3,5]$.

Les résultats généraux sont les suivants : l'onde lumineuse incidente excite dans la sphère diffringente des vibrations appartenant à une infinité de modes multipolaires électriques et magnétiques, la somme de leurs rayonnements constitue le champ diffracté. L'amplitude et la phase avec laquelle ces modes sont excités sont données par l'expression des coefficients de diffraction :

$$
\begin{aligned}
& a_{m}(\alpha, n)=(-1)^{m+1 / 2} \frac{S_{m}^{\prime}(\beta) S_{m}(\alpha)-n S_{m}^{\prime}(\alpha) S_{m}(\beta)}{S_{m}^{\prime}(\beta) \Phi_{m}(\alpha)-n \Phi_{m}^{\prime}(\alpha) S_{m}(\beta)} \\
& b_{m}(\alpha, b)=(-1)^{m+3 / 2} \frac{n S_{m}^{\prime}(\beta) S_{m}(\alpha)-S_{m}^{\prime}(\alpha) S_{m}(\beta)}{n S_{m}^{\prime}(\beta) \Phi_{m}(\alpha)-\Phi_{m}^{\prime}(\alpha) S_{m}(\beta)}
\end{aligned}
$$

Dans ces formules, $m$ est le rang du multipôle, $\lambda$ est la longueur d'onde de la lumière incidente dans le milieu extérieur, $a$ est le rayon de la sphère, $\alpha=2 \pi a / \lambda$ est le paramètre fondamental définissant cette dernière, $\beta=n \alpha, n$ est l'indice de réfraction de la matière constituant la sphère par rapport au milieu extérieur. C'est un nombre réel ou complexe selon que cette matière est transparente ou absorbante. Il est imaginaire pur si la sphère est parfaitement réfléchissante; le milieu extérieur est toujours supposé transparent.

La variable $z$ représentant $\alpha$ ou $\beta, S_{m}(z)$ et $C_{m}(z)$ sont les fonctions de Riccatti-Bessel définies par:

$S_{m}(z)=\sqrt{\frac{\pi z}{2}} J_{m+1 / 2}(z), \quad C_{m}(z)=\sqrt{\frac{\pi z}{2}} J_{-m-1 / 2}(z)$

où $J_{m+1 / 2}$ et $J_{-m-1 / 2}$ sont les fonctions de Bessel d'ordre $m+1 / 2$. Les dérivées $S_{m}^{\prime}(z)$ et $C_{m}^{\prime}(z)$ doivent être prises par rapport à $z$. On a posé de plus :

$$
\Phi_{m}(z)=S_{m}(z)+i C_{m}(z) .
$$

Toutes les grandeurs physiques relatives à la diffraction par les sphères sont données par des développements en série, où apparaissent les coef- ficients de diffraction. Nous renvoyons aux ouvrages cités en référence pour leur formulation et nous nous bornons à indiquer que l'expression du coefficient de diffusion est donnée par :

$$
K(\alpha, n)=\frac{2}{\alpha^{2}} \sum_{1}^{\infty}(2 m+1)\left[\left|a_{m}\right|^{2}+\left|b_{m}\right|^{2}\right] .
$$

Nouvelle méthode de calcul des amplitudes d'excitation des modes multipolaires. - Nous allons établir une propriété des coefficients de diffraction qui nous permettra de simplifier grandement les calculs. Considérons les expressions $(1,2)$ des coefficients de diffraction et posons :

$$
\left\{\begin{array}{l}
A=S_{m}^{\prime}(\beta) S_{m}(\alpha)-n S_{m}^{\prime}(\alpha) S_{m}(\beta) \\
B=S_{m}^{\prime}(\beta) C_{m}(\alpha)-n C_{m}^{\prime}(\alpha) S_{m}(\beta)
\end{array}\right.
$$

l'expression (1) se simplifie en :

$$
a_{m}(\alpha, n)=(-1)^{m+1 / 2} \frac{A}{A+i B} .
$$

En séparant les parties réelles et imaginaires, on obtient compte tenu de (4):

$$
\begin{aligned}
& a_{m}(\alpha, n)=R\left[a_{m}(\alpha, n)\right] \\
& \quad+i I\left[a_{m}(\alpha, n)\right]=(-1)^{m}\left[\frac{A B}{A^{2}+B^{2}}+i \frac{A^{2}}{A^{2}+B^{2}}\right] .
\end{aligned}
$$

Remarquons d'autre part que :

$$
\begin{aligned}
\left|a_{m}(\alpha, n)\right|^{2} & =\frac{A^{2} B^{2}}{\left(A^{2}+B^{2}\right)^{2}} \\
& +\frac{A^{4}}{\left(A^{2}+B^{2}\right)^{2}}=\frac{A^{2}}{A^{2}+B^{2}}=\left|I\left[a_{m}(\alpha, n)\right]\right| .
\end{aligned}
$$

La forme analogue du terme $b_{m}$ montre que le même résultat est valable également pour ce terme. Nous pouvons donc énoncer le théorème suivant :

" L'amplitude d'excitation d'un mode multipolaire est égale à la valeur absolue de la racine carrée de la partie imaginaire du coefficient de diffraction correspondant. ")

Comme conséquence, l'expression du coefficient de diffusion devient :

$$
K(\alpha, n)=\frac{2}{\alpha^{2}} \sum_{1}^{\infty}(2 m+1)\left\{\left|I\left[a_{m}(\alpha, n)\right]\right|+\left|I\left[b_{m}(\alpha, n)\right]\right|\right\} .
$$

Les diverses tables donnant directement les parties réelles et imaginaires des coefficients de diffraction, on peut juger de l'avantage qu'apporte ce nouveau résultat pour le calcul numérique des phénomènes. En effet, le théorème que nous venons de démontrer permet de déduire à simple lecture l'intensité d'excitation des modes, alors que le calcul classique aurait exigé la détermination des carrés de chaque terme, puis l'extraction de la racine de leur somme. L'opération eut été longue, l'étude d'un seul mode nécessitant le calcul de plusieurs centaines de points. 
Remarque. - Rappelons une propriété intéressante obtenue par Van de Hulst à l'aide de raisonnements analogues. Il établit que le lieu des affixes des points représentatifs des coefficients de dif-

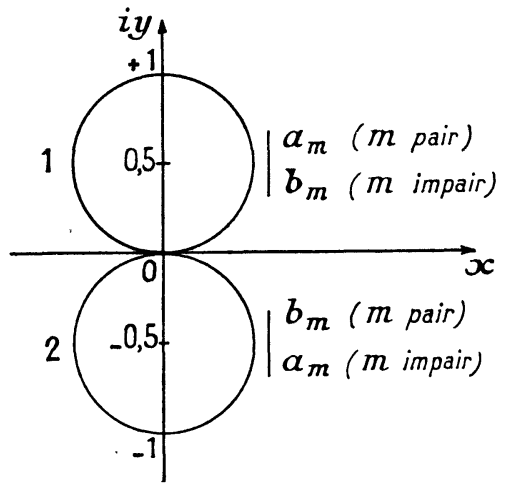

Fig. 3. - Lieu des affixes des coefficients de diffraction.

fraction est un cercle. Les notations employées ici étant légèrement différentes, ce résultat se transforme comme suit : les affixes des coefficients de diffraction se répartissent sur deux cercles de centres $\pm 0,5 i$, de diamètre 1 ( $f$ g. 3 ). Les termes $a_{m}$ sont situés sur le cercle (1) si $m$ est pair, sur le cercle (2) si $m$ est impair. Pour les termes $b_{m}$, le résultat est opposé : cercle (1) pour $m$ impair, cercle (2) pour $m$ pair.

Étude de l'excitation des modes multipolaires électriques et magnétiques. - $a$ ) TeGHNIqUE DES CALCULS. - A l'aide de la propriété que nous avons établie au paragraphe précédent, nous avons déterminé, dans le cas de $n=1,44$, pour chaque valeur des coefficients calculés par Penndorf, les intensités des 34 premiers termes multipolaires électriques et magnétiques. Les calculs sont effectués par incréments de 0,1 $\alpha$. Rappelons que seule la valeur locale de l'intensité d'excitation est déterminée, mais non le sens de sa variation avec $\alpha$.

b) Résultats de L'examen des courbes D'EXcitation DES MODES. - Sur les figures, nous portons en abscisses, le paramètre $\alpha$, en ordonnées, l'intensité d'excitation des modes. Pour la clarté $\mathrm{du}$ dessin, nous ne représentons (traits fins) que les 10 premiers modes électriques $a_{m}$ (fig. 4) et magnétiques $b_{m}$ (fig. 5). Nous superposons à chacun des réseaux la courbe de diffusion $K(\alpha, 1,44)$ (gros trait).

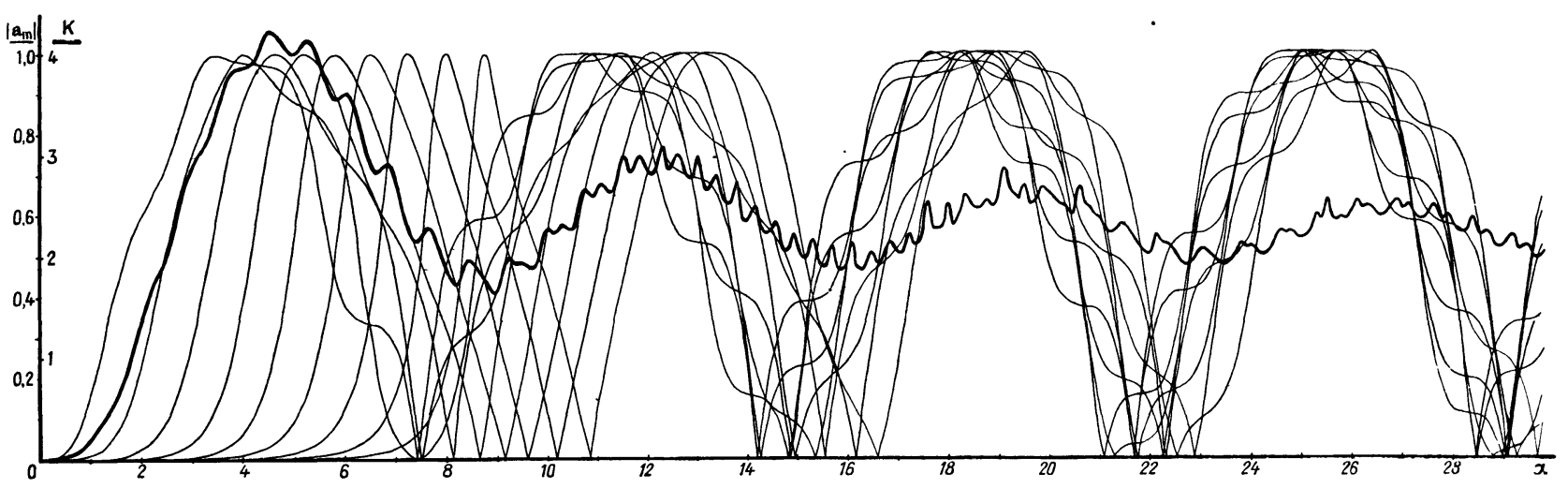

Fig. 4. - Intensités des premiers modes multipolaires électriques (traits fins) et courbe de diffusion $K(\alpha, n)$ (gros trait).

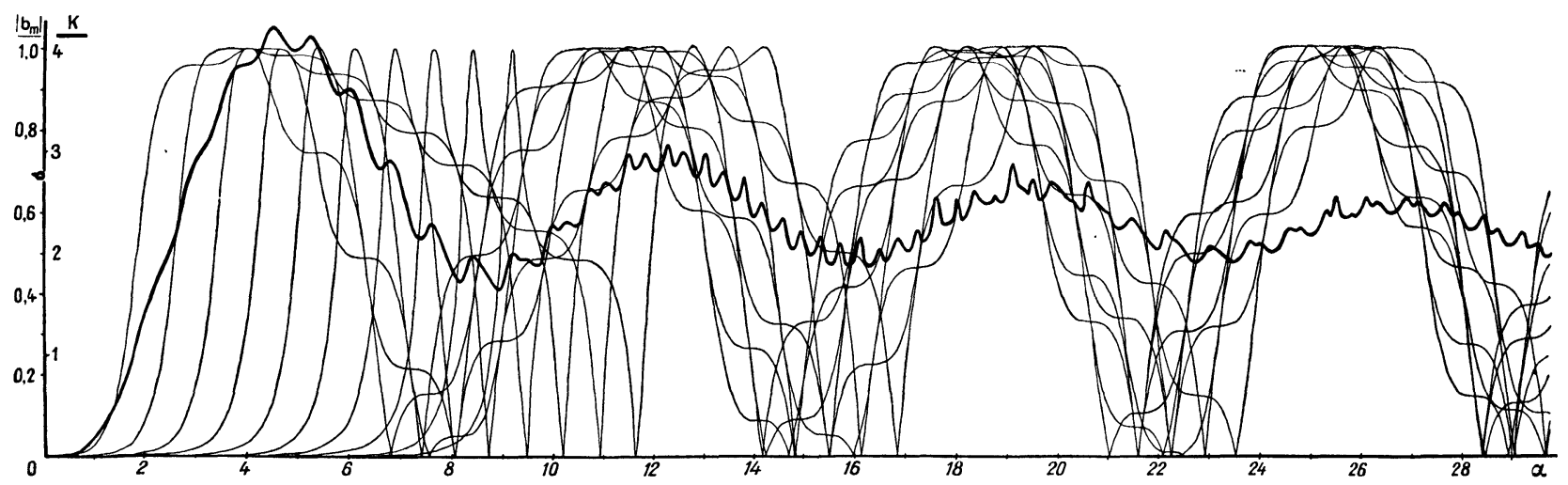

Fig. 5. - Intensités des premiers modes multipolaires magnétiques (traits fins) et courbe de diffusion $K(\alpha, n)$ (gros trait). 
On constate que chaque courbe est constituée d'une série de maxima généralement flous ayant pour borne supérieure 1, séparés par des minima nuls. A ces oscillations amples se superposent des ondulations mineures qui nous paraissent difficiles à interpréter. Nous ne le tenterons pas, car ceci ne présente aucun intérêt pour la suite. Les caractères essentiels qui se dégagent de l'examen de ces graphiques sont les suivants :

1) Le comportement des modes électriques et magnétiques de même rang $m$ est semblable.

2) La largeur du premier maximum décroît régulièrement quand $m$ croît et finit, pour les grandes valeurs de $m$, par devenir très inférieure à $0,1 \alpha$. Il disparaît donc de la tabulation, comme le montrent les valeurs suivantes relatives au $30^{\mathrm{e}}$ mode magnétique

$$
\begin{array}{rr}
\alpha=24,1 & I\left(b_{m}\right)=0,0065 \\
24,2 & 0,0136 \\
24,3 & 0,0425 \\
24,4 & 0,0292 \\
24,5 & 0,0145
\end{array}
$$

Le premier maximum, égal à 1 , est situé entre 24,3 et 24,4 , il a échappé à l'analyse.

3) Au delà de $m=10$, le second maximum s'affine à son tour. A titre indicatif, nous donnons (fig. 6) la courbe d'excitation d'un mode supérieur : $30 \mathrm{e}$ magnétique.

4) Les premiers maxima des modes magnétiques sont plus fins que les maxima électriques de même rang.

5) Les cinq premiers maxima électriques et magnétiques, assez flous, se groupent au voisinage de $\alpha=4,5$ : il en résulte la première ondulation principale de la courbe de diffusion.
6) Pour $\alpha>6$ (fig. 5), les indentations secondaires de la courbe de diffusion correspondent aux premiers maxima des modes magnétiques des divers ordres.

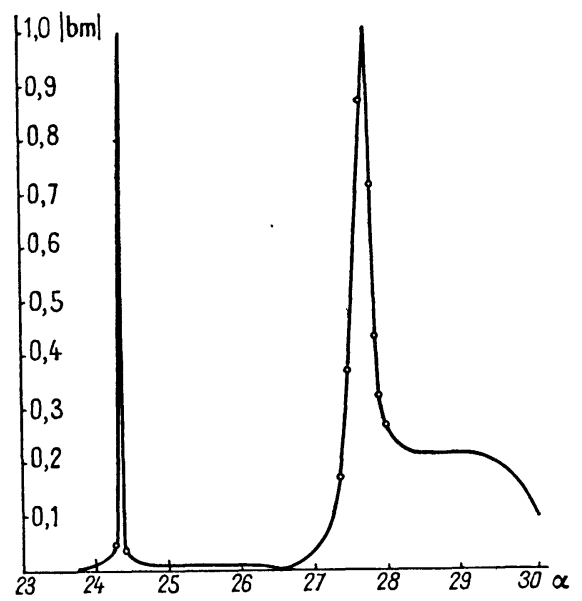

FIG. 6. - Intensité du $30^{\mathrm{e}}$ mode magnétique.

Interprétation des courbes de diffusion. - Pour préciser le résultat que nous venons de signaler, nous avons tracé, sur un même graphique avec les mêmes coordonnées que dans le paragraphe précédent, les premiers maxima des modes magnétiques (tirets) et électriques (pointillés), ainsi que, superposée à ces réseaux, la courbe de diffusion (trait plein). Pour faciliter l'exposé, nous divisons en trois régions l'intervalle de variation de $\alpha$.

a) 1 re RÉGION $\alpha<18$ (fig. 7). - 1) Pour $\alpha>6$, à chaque premier maximum des modes magné-

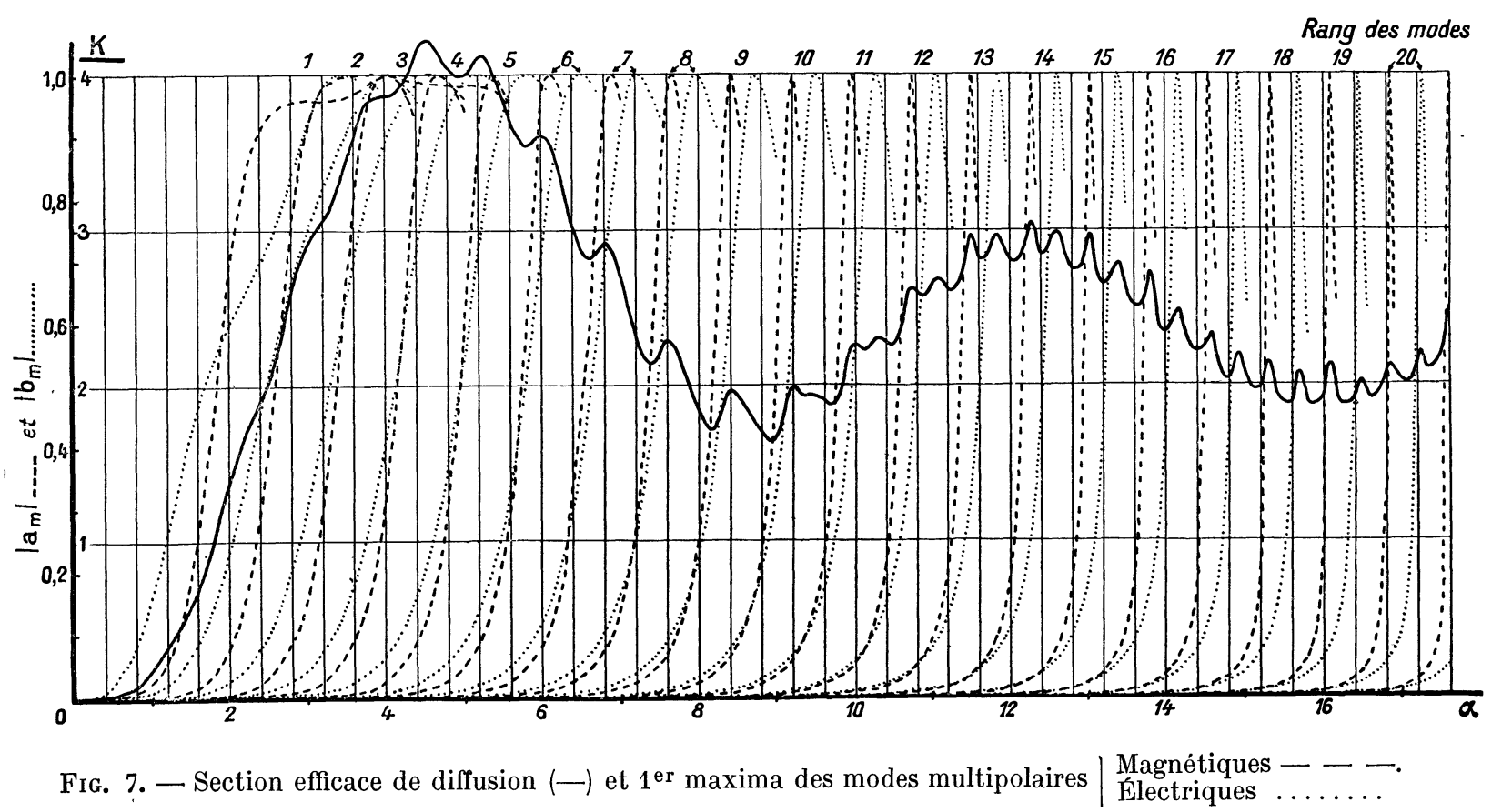


tiques successifs correspond une indentation de $K(\alpha, n)$.

2) Pour $\alpha>10$, une nouvelle série d'indentations se rapportant au premier maximum des modes électriques des divers ordres, vient s'intercaler entre les précédentes. On observe que l'action d'un mode sur la courbe de diffusion est d'autant plus marquée que le maximum d'excitation correspondant est plus aigu : c'est pourquoi les composantes électriques n'agissent que pour des ordres assez élevés.

3) Pour $\alpha<6$, l'origine des indentations semble difficile à élucider. Tenant compte de l'orthogonalité des modes et de leur action purement additive, nous proposons l'interprétation suivante :

$5^{\mathrm{e}}$ indentation: $(\alpha=5,3)$, superposition des $4^{\mathrm{e}}$ modes électrique et $5^{\mathrm{e}}$ magnétique.

$4^{\mathrm{e}}$ indentation: $(\alpha=4,5)$, superposition des $3^{\mathrm{e}}$ modes électrique et $4^{\mathrm{e}}$ magnétique.

Les trois précédentes, réduites à des inflexions, résultent de la superposition des trois premiers modes des deux genres. Il ne semble pas possible de préciser davantage.

b) $2^{\mathrm{e}}$ RÉGION $18<\alpha<23$ (fig. 8). - Les indentations de $K(\alpha, n)$ correspondent encore aux pre-

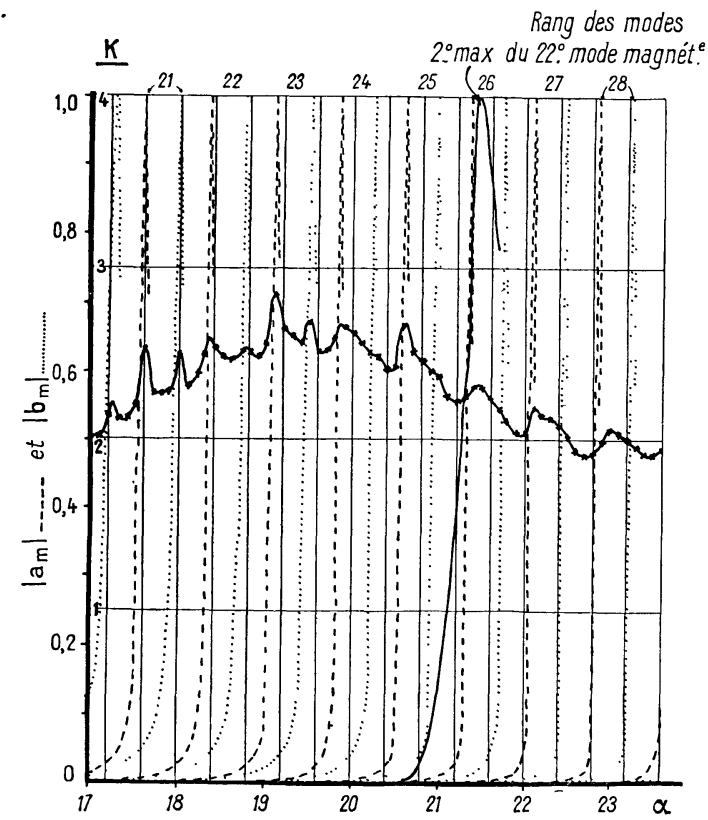

FIG. 8. - Section efficace de diffusion (-) et $1^{\mathrm{er}}$ maxima des modes multipolaires $\mid \begin{aligned} & \text { Magnétiques }-\ldots \ldots \\ & \text { Électriques } \ldots \ldots\end{aligned}$

miers maxima des modes successifs, mais leurs amplitudes sont irrégulières, certaines ont même disparu. Il ne s'agit là que d'une apparence, due à un intervalle trop large des points calculés. En effet, si l'on porte sur la courbe de diffusion les points tabulés, on observe que, selon qu'ils coïn- cident ou non les avec maxima d'excitation, l'ondulation est forte ou faible. Remarquons d'autre part qu'une des indentations $(\alpha=21,4)$ correspond, non plus au premier, mais au second maximum d'excitation du $22^{\mathrm{e}}$ mode magnétique.

c) 3e RÉGION $23<\alpha<30$ (fig. 9). - Les indentations sont faibles et coincident cette fois avec les

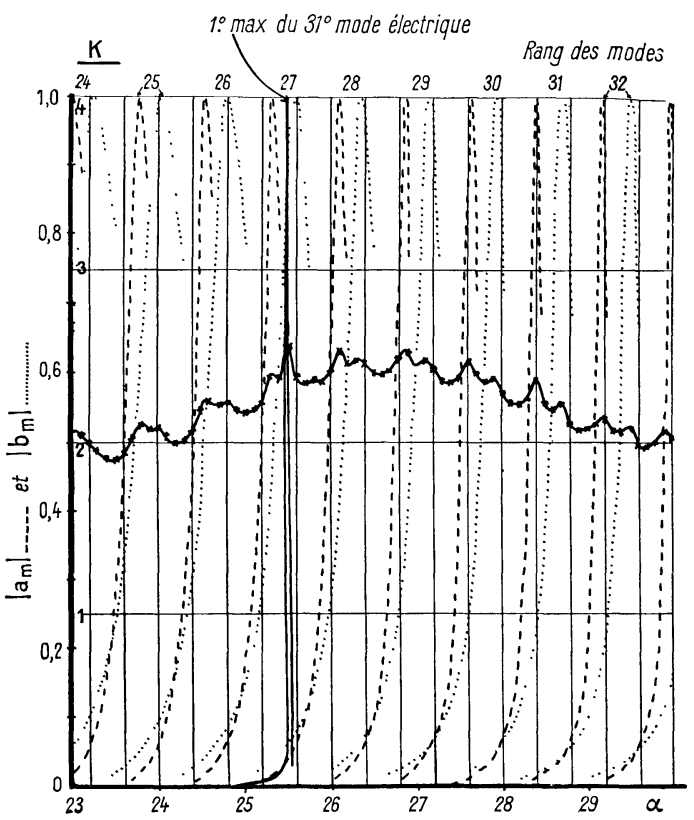

Fig. 9. - Section efficace de diffusion (-) et $2^{\mathrm{e}}$ maxima des modes multipolaires $\mid \begin{aligned} & \text { Magnétiques }-\ldots \text { Électriques } \ldots . . . \\ & \text {. }\end{aligned}$

seconds maxima des différents modes. Celles qui sont dues aux premiers maxima d'excitation ont disparu, à l'exception du $31 \mathrm{e}$ électrique. Nous admettons, comme pour la région précédente, que cette anomalie est due à ce que les pointes des premiers maxima sont si fines qu'elles échappent à l'analyse.

Pour corroborer notre hypothèse, nous avons indiqué, dans le tableau ci-dessous, la plus grande valeur numérique d'amplitude d'excitation tabulée au voisinage des premiers maxima contenus dans cette région.

\begin{tabular}{|c|c|c|c|c|c|}
\hline $\begin{array}{c}\text { MODE } \\
\text { ÉLEGTRIQUE }\end{array}$ & $\alpha_{\mathrm{m}}$ & $\begin{array}{l}\text { AMPLI- } \\
\text { TUDE }\end{array}$ & $\begin{array}{c}\text { MODE } \\
\text { MAGNÉTIQUE }\end{array}$ & $\alpha_{\mathrm{m}}$ & $\begin{array}{l}\text { AMPLI- } \\
\text { TUDE }\end{array}$ \\
\hline 28 & 23,3 & 0,1700 & 28 & 22,8 & 0,0803 \\
\hline 29 & 24,0 & 0,2748 & 29 & 23,6 & 0,1503 \\
\hline 30 & 24,8 & 0,0774 & 30 & 24,3 & 0,0425 \\
\hline 31 & 25,5 & 0,9289 & 31 & 25,1 & 0,0444 \\
\hline 32 & 26,2 & 0,0412 & 32 & 25,8 & 0,4309 \\
\hline 33 & 27,0 & 0,0794 & 33 & 26,5 & 0,0209 \\
\hline 34 & 27,7 & 0,0478 & 34 & 27,3 & 0,0305 \\
\hline 35 & 28,5 & 0,0200 & 35 & 28,0 & 0,0389 \\
\hline 36 & 29,2 & 0,1463 & 36 & 28,8 & 0,0080 \\
\hline 37 & 29,9 & 0,0136 & 37 & 29,5 & 0,0168 \\
\hline
\end{tabular}


Positions des premiers maxima des modes multipolaires. - Il serait trop long de déterminer directement les positions des premiers maxima des modes multipolaires à partir des équations (1) et (2) qui sont très complexes. Seul, un examen attentif des amplitudes tabulées permet de les localiser à $0,1 \alpha$ près.

Pour préciser comment varie leur position en fonction de $\alpha$, nous portons, en abscisses (fig. 10),

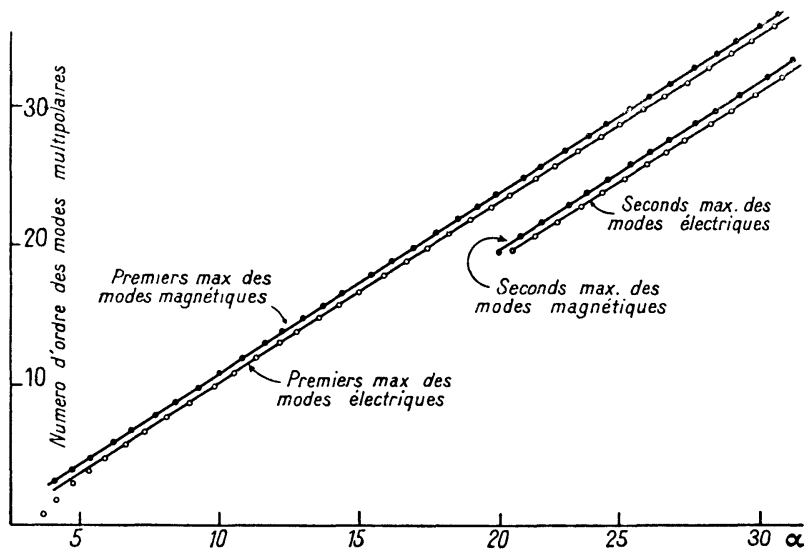

FIg. 10. - Position des premiers et seconds maxima des modes multipolaires électriques et magnétiques.

les valeurs de $\alpha$ se rapportant successivement aux deux premiers maxima des modes électriques et magnétiques, en ordonnées, les rangs des multipôles correspondants. A l'exception des tous premiers, les points ainsi obtenus se groupent sur quatre droites sensiblement parallèles. En d'autres termes, les maxima de même origine sont à peu près équidistants.

On peut représenter les valeurs de $\alpha$ correspondant à ces maxima par les formules approchées suivantes :

$$
\begin{array}{ll}
1^{\mathrm{er}} \max . \text { électrique } & \alpha=0,764 \mathrm{~m}+1,86 \\
2^{\mathrm{e}} \max . \text { électrique } & \alpha=0,776 \mathrm{~m}+4,76 \\
1^{\mathrm{er}} \max . \text { magnétique } & \alpha=0,756 \mathrm{~m}+1,65 \\
2^{\mathrm{e}} \max . \text { magnétique } & \alpha=0,765 \mathrm{~m}+4,67 .
\end{array}
$$

Dans tout l'intervalle $10<\alpha<30$, l'écart entre les valeurs déduites des tables et celles données par ces formules est inférieur à une unité du premier ordre décimal. Il croît légèrement en dehors de cet intervalle. Son sens prouve que les courbes possèdent une légère concavité tournée vers le bas, que l'on peut négliger en première approximation.

Détermination quantitative de l'amplitude des indentations. - a) LE MAXIMUM D'EXcitation PEUT ÊTRE CONSIDÉRÉ COMME INFINIMENT ÉTROIT. - On peut déterminer l'amplitude des indentations par le raisonnement suivant : dans l'expression (10) qui représente le coefficient de diffusion, tous les termes sont positifs et additifs. D'autre part, nous avons montré plus haut que les premiers maxima des ordres multipolaires supérieurs sont constitués par une pointe très aiguë et isolée, on peut donc séparer dans la série le terme qui atteint la valeur 1. Il n'agit que localement puisqu'il devient négligeable dès que l'on s'écarte de la valeur de $\alpha$ correspondant à son maximum. En conséquence, l'ensemble des autres termes donne la courbe moyenne et le terme résonnant l'indentation.

Ce raisonnement donne une approximation correcte, dès que la pointe est assez aiguë (grandes valeurs de $\alpha$ ). La contribution du terme de rang $m$ est alors :

$$
A(m)=\frac{2}{\alpha^{2}}(2 m+1) .
$$

Le premier maximum du terme $a_{31}$

$$
I\left(a_{m}\right)=0,929
$$

coïncide presque avec l'un des points calculés et permet une vérification de ce résultat. La hauteur de l'indentation qui $\in \mathrm{n}$ résulte est :

$$
\begin{aligned}
& \Delta K \text { calculé }=0,194 \\
& \Delta K \text { mesuré }=0,18 .
\end{aligned}
$$

Si l'on extrapôle la loi linéaire donnant les positions des maxima, on constate que les amplitudes diminuent selon une loi en $1 / \mathrm{m}$ lorsque $m$ croît indéfiniment.

b) Le MaXimum D'EXcitation a UNe Largeur NON NÉGLIGEABLE. - Dans ce cas, l'amplitude de l'indentation dépend del'acuité du maximum d'excitation du multipôle correspondant. Nous n'avons pas effectué les calculs qui s'y rapportent parce que, dans l'intervalle où ils s'imposent ( $f i g .8)$ les indentations sont assez larges pour être bien définies par les tables.

Application : Forme correcte de la courbe de diffusion dans l'intervalle $23<\alpha<30$. - Nous avons noté plus haut que, dans cet intervalle les indentations dues aux premiers maxima des modes avaient disparu à la tabulation. Nous avons calculé, selon notre méthode, leurs amplitudes et nous les avons portées sur la courbe moyenne de diffusion (fig. 11).

La structure secondaire désordonnée qui avait étonné différents auteurs (3) s'est régularisée. La succession des indentations simples et dédoublées de la courbe de Penndorf a disparu.

La nouvelle structure est formée par la superposition de quatre séries d'indentations correspondant aux deux premiers maxima des modes électriques et magnétiques. L'évolution de chaque série est la suivante : les ondulations d'abord larges et peu intenses s'affinent en même temps que leur amplitude croît. Cette dernière passe par un maximum puis décroît lentement tandis que la largeur de l'indentation diminue constamment. La pre- 
mière série qui apparaît est celle des 1 ers maxima magnétiques, puis, celle des 1 ers maxima électriques vient s'y intercaler. Pour une valeur de $\alpha>22$, apparaissent successivement les 2 es maxima magnétiques et électriques. Comme l'inter-
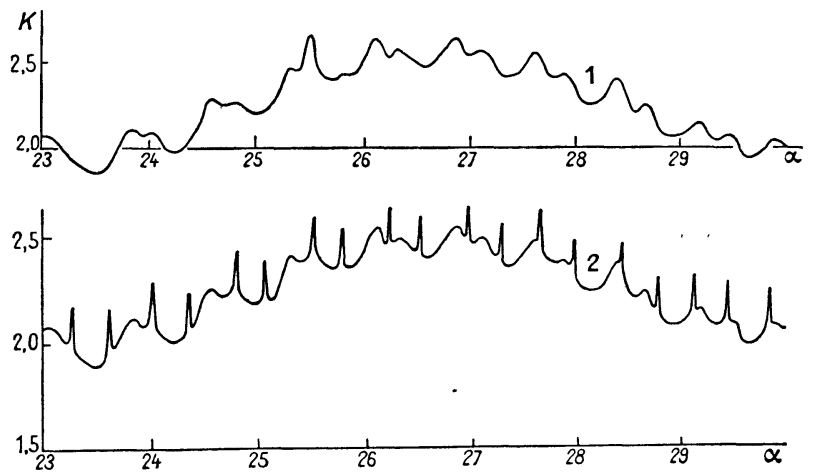

FIG. 11. - Structure détaillée de la courbe de diffusion. (1) D'après les tables de Penndorf - courbe très altérée par une analyse numérique insuffisante.

(2) Courbe correcte. - Détails restitués par notre méthode.

valle entre in dentations est le même dans toutes les séries, on obtient l'aspect de la figure 11. Si l'on extrapôle au delà de $\alpha=30$, le même processus doit continuer avec apparition de nouvelles indentations correspondant aux $3^{\text {es }}$ puis aux $n^{\text {iemes }}$ maxima des deux ordres. La structure ultime est étrangement complexe.
Signalons que sans l'interprètation nouvelle que nous avons donnée, il eut été très difficile d'obtenir ce résultat. Il eut fallu effectuer les calculs numériques à intervalles très serrés, le travail eut été considérable et surtout l'extrapolation impossible.

Conclusion. - La structure détaillée de la courbe de diffusion de la lumière par les particules sphériques transparentes est due aux premiers maxima des modes multipolaires successifs. L'intensité des indentations caractéristiques de cette structure peut se déterminer en séparant l'action du terme multipolaire qui passe par un maximum de celle de l'ensemble de tous les autres termes.

Nous avons, par une méthode nouvelle, reconstitué l'aspect de la structure détaillée de la courbe de diffusion correspondant aux valeurs de $\alpha$ inférieures à 30. Elle est constituée d'une succession complexe d'indentations régulièrement disposées dont certaines sont très aiguës, leurs positions sont données par des lois sensiblement linéaires. Nous montrons comment l'extrapolation de ces résultats permet de prévoir la structure de la courbe pour les grosses sphères.

Nous tenons à exprimer notre gratitude à $\mathrm{M}$. le pr Grivet pour l'intérêt qu'il a porté à cette étude. Nous remercions M. D€ws, du Geophysical Research Directorate de Cambridge (U. S. A.), qui a rendu ce travail possible en nous procurant gracieusement un exemplaire des tables de Penndorf.

Manuscrit reçu le 4 avril 1958.

\section{BIBLIOGRAPHIE}

[1] Penndorf (R.) et Goldberg (B.), "New Tables of Mie scattering function for spherical particles. " Geophysical Research Paper no 45-Geophysics Research Directorate-Air force Cambridge Research Center-Cambridge, Mass., U. S. A., 1956.

[2] GoldBerg (B.), "New computations of the Mie scattering functions for spherical particles. "J. Opt. Soc. Amer., 1953, 43, 1221.
[3] Van de Hulst (H. C.), " Light scattering by small particles ", New York, Wiley \& Sons, 1957.

[4] MIE (G.), "Beitrage zür Optik trüber Medien, speziell Kolloidaler Metallosungen. "Ann. der Physik (4), 1908, 25, 377-445.

[5] MÉveL (J.), "Contribution à l'étude de le diffraction des ondes électromagnétiques par les sphères. "Thèse à paraître, Rennes, 1957. 